\title{
A Renewable Natural Bioactive Polysaccharide: An Overview to Chitosan
}

\author{
Büşra Ayhan, Nevin Şanlier \\ Department of Nutrition and Dietetics, Faculty of Health Sciences, Gazi University, Ankara, Turkey
}

Email address:

busraayhan989@gmail.com (B. Ayhan),nevintekgul@gmail.com (N. Şanlıer)

\section{To cite this article:}

Büşra Ayhan, Nevin Şanlier. A Renewable Natural Bioactive Polysaccharide: An Overview to Chitosan. International Journal of Nutrition and Food Sciences. Vol. 4, No. 5, 2015, pp. 541-548. doi: 10.11648/j.ijnfs.20150405.15

\begin{abstract}
Chitin is a polysaccharide, which can be found in the structures of some insects, yeasts and fungi in nature. Chitosan is a compound, which is obtained by de - acetylation of chitin. Apart from that, there is chitosan naturally on the cell walls of some fungi, in yeasts and protozoa and in the outer shells of crustaceans. Chitosan is a molecule, which is frequently used in health field and food industry. It is frequently used in bioactive packing especially for its antibacterial effect. In addition, it is used in many areas such as waste treatment for its antioxidant feature. Areas of use of chitosan are expanding because it is an edible agent and because of its low level of toxic activity, and new areas of use are discovered everyday in good and health sectors.
\end{abstract}

Keywords: Chitosan, Chitin, Antimicrobial

\section{Introduction}

Chitin is the most available polysaccharide in nature after cellulose and it is generally fount at the outer shells of crustaceans. In addition, it can also be found in the structures of various insects, larvivorous organisms, yeasts - fungi and mushroom in various amounts. Chitin constitutes $45 \%$ of cell wall of Aspergillus niger and Mucor rouxii and $25 \%$ of cell wall of Penicillium notatum [1]. Although chitosan in essence is a compound derived from chitin, it can naturally be found on the cell walls of Zygomycetes class fungi, Chlorella sp. Green algae, yeasts and protozoa, cuticula layers of insects and especially on the outer shells of crustaceans [2].

\section{Structure and Features of Chitosan}

Chitin is a polysaccharide, which is formed of as the result of binding of 2 - acetamide -2 deoxy - D - glucopyronose and 2 - amino -2 deoxy $-\mathrm{D}-$ glucopyronose units with $\beta$ (1-4) bond [3]. Chitosan is a compound, which commercially obtained by applying chemical reaction, which include de mineralization, de - proteinization, de - clorization, and de acetylation processes [4]. Chitin $70 \%$ de- astyle chitosan solution is obtained after de- asetylation of chitin for $1-3$ hours in $40 \%$ sodium hydroxide solution under $120^{\circ} \mathrm{C}$. The purpose of that application is to obtain pure chitin after removing protein, mineral and pigment structures in chitin. Chitosan compound is obtained as the result of those de acetylation applications [5].

Chitin has a chemical structure similar to that of cellulose [poly- $\beta-(1-4)-N$-acetyl-D-glucosamine]. However, an acetylamide group is bound instead of hydroxyl group in each monomer in chitosan. Average molecule weight of chitosan changes from $1 \times 10^{5}$ to $5 \times 10^{5} \mathrm{Da}$ [6].
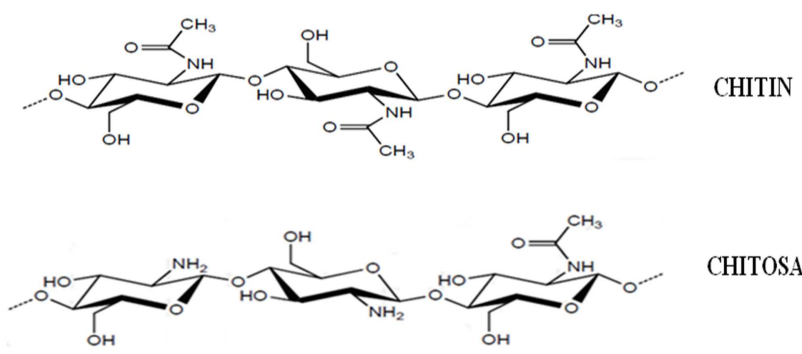

CHITOSAN

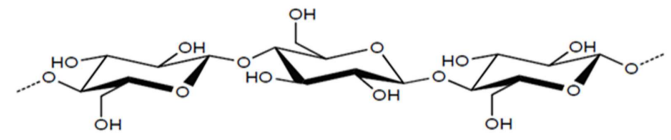

CELlulose

Figure 1. Chemical structures of chitin, chitosan and cellulose [7].

The two most important chemical features of chitosan are its se - acetylation degree and its molecule weight. De - 
acetylation phase, which is applied to obtain chitosan, is conducted by using concentrated alkaline solution under high temperatures in a specified period of time. De - acetylation degree is effective on every physico - chemical features, such as molecule weight, viscosity and solubility. As the de acetylation degree increases. So does the solubility of the compound. The changes in the de- acetylation conditions that are applied to chitosan affect the molecule weight of the chitosan as well. The solved oxygen in the environment during de - acetylation causes the fragmentation of the structure and thus decreases in the weight of the molecule. It is among the factors which decrease the weight of the molecule in the very high temperatures, at which de acetylation is applied. Molecule weight of the chitosan is effective on its antibacterial feature [8].

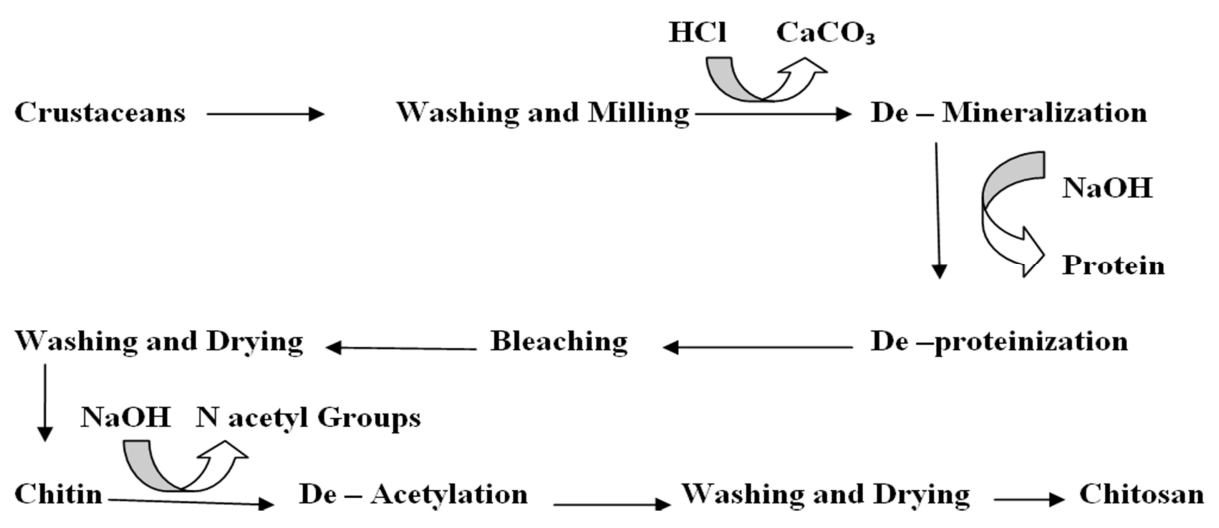

Figure 2. Chemical process of Chitosan production [9].

Chitosan is a compound, which is not soluble in many organic solutions and water with neutral $\mathrm{pH}$. However, it can be dissolved in organic acid solutions such as acetic acid, formic acid, tartaric acid, valeric acid, glycolic acid, and citric acid and also in diluted inorganic acid solutions such as hydrochloric acid and sulfuric acid. The fact that chitosan is not soluble in water constitutes a significant impediment against it can have an extensive antibacterial effect. In the studies conducted in recent years in order to develop that feature of chitosan, its solubility on water was tried to be increased by subjecting it to de- polymerization in half [10, $11]$.

\section{Chitosan's Usage in Food Industry}

\subsection{Its Antimicrobial Feature}

Start of the studies on chitosan in modern ages goes back to 1990 s and its first feature, which was discovered, was its antimicrobial feature. Chitosan and chitosan derivatives show antimicrobial activity in a wide spectrum on human pathogens and on bacteria, which cause food borne intoxication. It was found out in the first studies on the antimicrobial activity of chitosan that it had an inactivating effect on both Graham positive and Graham negative bacteria in a wide spectrum [2, 12]. In the further studies conducted in the following years, six different mechanisms regarding the antimicrobial feature of chitosan were explained:

- Positive loaded amine groups of chitosan interact with negative loaded microbial cell membranes. That enables compounds with protein and other inner cell compounds to leak out.

- Chitosan blocks active parts of various enzymes and causes enzyme inhibition and activates some protective systems by working as water bound agent in host tissue.

- With its binding feature, chitosan binds some metals to itself and that enables to suppress the toxin production and stopping microbial growth in the cell.

- Chitosan with high molecule weight forms a layer, which does not allow material transfer, on the surface of the cell. In that way, it changes the cell transitivity and prevent food entry in the cell.

- Chitosan with low molecule weight interferes with the penetration of microorganism through stazole in order to bind DNA and with the mRNA and protein synthesis.

- Chitosan breaks the physiologic activities such as adsorbtion in the microorganism cell and flocculation of electronegative substances and causes the death of the microorganisms $[1,12,13]$.

In addition to them, it is important that chitosan is soluble only in acidic environment. For that reason, effects of $\mathrm{pH}$ on the microorganisms should be considered while talking about the antimicrobial feature of chitosan. In other words, $\mathrm{pH}$ and chitosan show synergist energy in antimicrobial activity [12].

Antimicrobial feature of chitosan is not the same for every bacterium. For that reason, while mentioning the activation of chitosan on the microorganisms, various parameters that have effect on that activation should be mentioned as well. Features, which rae special to microorganisms (structure special to microorganism, age of microorganism, etc.), features, which are special to chitosan (intenseness of positive load of chitosan, Protonation levels of amine groups, molecule weight, concentration, its state as solved in water or as in solid particulars, etc.) or environmental factors (ionic force of test environment, $\mathrm{pH}$, temperature, period of exposure, etc) are the situations which are effective on the antimicrobial activation of chitosan [13]. In a study, with which the effects of chitosanium acetate films on microorganisms were examined, it was observed that growth 
of L. monocytogenes, Salmonella and S. aureus completely stopped at $37{ }^{\circ} \mathrm{C}$ when they were covered with $80 \mathrm{mg}$ chitosan film [14]. According to the results of another study conducted, the amounts of concentrations required for chitosan and its derivatives to show their inhibitor effects according to microorganism types are given in Table 1 (14):

Table 1. The amounts of concentrations required for chitosan and its derivatives to show their inhibitor effects [15].

\begin{tabular}{lllll}
\hline Microorganism & Chitin & Chitosan & N-acetyl chito - oligosaccharide & Chito - oligosaccharide \\
\hline Escherichia coli & 0.01 & 0.01 & 0.003 & 0.003 \\
Pseudomonas aeruginosa & $>0.1$ & 0.05 & 0.003 & 0.003 \\
Staphylococcus aureus ATCC 43300 & 0.03 & 0.03 & 0.003 & 0.003 \\
Staphylococcus aureus ATCC 25923 & 0.03 & 0.03 & 0.003 & 0.003 \\
Salmonella typhimurium & $>0.1$ & $>0.1$ & 0.003 & 0.003 \\
Bacillus subtilis & 0.03 & 0.01 & 0.003 & 0.003 \\
Bacillus cereus & 0.03 & 0.01 & 0.003 & 0.003 \\
Vibrio cholerae & 0.01 & 0.01 & 0.003 & 0.003 \\
Shigella dysenteriae & 0.01 & 0.01 & 0.003 & 0.003 \\
Enterobacter agglomerans & 0.01 & 0.01 & 0.003 & 0.003 \\
Prevotella melaninogenica & $>0.1$ & 0.01 & 0.003 & 0.003 \\
Bacteroides fragilis & 0.006 & 0.006 & 0.003 & 0.003 \\
\hline
\end{tabular}

\subsection{Its Feature of Forming into Film and Its Usage in Bioactive Packing}

Packing is of great importance for foods with short live such as sea food. Although traditional packing methods are used for packing those products, alternative packing materials have been sought because of the known negative effects of traditional methods both on the food and on environment. As it is known that packing materials have effect on costs and causes increase in the sale price of the final product, the alternative packing materials are required as low cost materials. In accordance with that requirement, production of edible bioactive packing materials has accelerated especially in the recent years. Chitosan is one of the materials, which is frequently used in the production of those bioactive materials. Chitosan films tend to show resistance against lipid diffusion and differential permeability of gasses. Because of this quality, it has a fine protection for oxygen, nitrogen and carbon dioxide permeability in the food. It is reported that chitosan forms a barrier between the food and the atmospheric gasses around and delays the lipid oxidation by slowing down the diffusion of oxygen in the food in that way [16]. In a study conducted on the usage of chitosan in bioactive packing, it was found out that chitosan packing enriched with cinnamon oil decreased the lipid oxidation and number of peroxide for 16 days [17]. Those findings were supported by other studies and it was found out that chitosan helped for protecting the qualities of food products during their storage [18]. In another study, the red pepper, which was stored 21 days at $7{ }^{\circ} \mathrm{C}$ at first and then 5 days at $20{ }^{\circ} \mathrm{C}$, was found out that did not have any changes in its ascorbic acid, phenolic agent and antioxidant contents when it was covered with chitosan [19]. Apart from all those positive effects, chitosan has significant inefficiency in terms of water resistance and vapor transfer. It was reported that chitosan inactivates thyrosinaze enzyme, which causes decay especially on fruit and vegetables, and increases their storage periods and shelf lives. It is stated that covering fruit and vegetables with chitosan decreases the oxygen pressure that would occur on food product and keeps the temperature under control by enabling moisture balance and protects especially the fruit against tarnishing because it prevents water loss. In addition to all those, it is also possible that it is used for delaying ripening for some vegetables. Besides, covering with chitosan film can be used for milk and meat products for protection purposes because of the preventing feature of chitosan on lipid oxidation. Chitosan can be used instead of sulfide group as browning inhibitor in recent years $[4,5]$.

\subsection{Its Usage in Waste Treatment}

Chitosan can be used as coagulant in order to remove the solid matters during some processes such as whey and milk wash water during poultry products and sea products processes. The level of decreasing the amount of solid matters and reducing solution blurriness of chitosan, which is used for waste treatment, changes depending on the amount of its usage. In addition, even the protein recovery levels of chitosan with different molecule weights and de - acetylation levels differs one from the other. Use of chitosan with alginate as a complex can be an alternative for the recovery of soluble proteins to be disposed as wastes and during the further and more expensive phases in commercial membrane applications and their restricted uses because of environmental pollution. The basis of that waste treatment mechanism, especially on the marine plants, of the chitosan is its positive load feature and that it can easily interact with negative loaded compounds such as proteins. In addition, hydroxyl groups of chitosan contribute to the precipitation of proteins in marine plants and other soluble solid matters [2].

\subsection{Its Contribution to Emulsion and Stabilization}

Chitosan has been seen to be an interest arising and promising emulsifier and stabilizer in recent years. It is reported that chitosan is a good emulsifier agent and makes a strong emulsion. It is thought that its emulsifying capacity comes from its heterogenic structure. D - Glucosamine, which is the basic structure of chitosan. Has a strong hydrophilic feature. In addition, $\mathrm{N}$ - acetyl residues, which are exposed to less de- acetylation, has hydrophobic features as well. Practically, chitosan is used for emulsion 
stabilization, especially under acidic conditions. Because of its negative loaded surface droplets, chitosan has an electrostatic attraction and it has an important role in enabling emulsion development stability in foods through electrostatic stabilization [9].

\section{Chitosan's Usage in Health Area}

\subsection{Its Antioxidant Feature}

Sea products are very precious food especially because they include eicosapentaenoic acid (EPA) and cocosahexaenoic acid (DHA), which cannot be produced in human body, and also known as "omega 3" fatty acid as essential fatty acid in high levels. However, those omega 3 fatty acids are very sensitive against oxidation reactions may be subject to loss of taste that may displease the consumers during their storage $[2,12]$.

In order to prevent the mentioned potential negative reactions that may happen on the sea products because of the increasing consumer interest to the fish and other sea products for their benefits, chitosan may be added to those products as a synthetic antioxidant. In a study where one of the traditional antioxidants and chitosan applied on the fished stored at $4{ }^{\circ} \mathrm{C}$ and the results were examined, $14 \mathrm{cP}$ chitosan was applied in $200 \mathrm{ppm}$ amount. At the end 8 days storage period, $52 \%$ decrease was observed in the reactive matters in the fish compared to the fish that chitosan was not applied and it was stated that this was a decrease in the similar level with the traditional antioxidant applications [20].

When its antioxidant feature is considered, it is thought that chitosans with different viscosity has different activities, cationic groups of chitosan would produce an inner molecule driving force when it is loaded and that would decrease the binding capacity in high level viscosity. And the difference in viscosity is thought to be arising from the acetylation degree or molecule weight of chitosan. It is reported that the oxidant binding capacity of chitosan, which is acetylated in high amounts, is lower. In addition, it is also reported that chitosan has a tighter structure as long as its molecule weight increases and its inner molecule hydrogen bounds are stronger. That situation decreases the activities of hydroxyl and amino groups of chitosan. As a result, it is thought that this fact may cause the active parts of the molecule having a limited antiocidant effect. It is stated that, as long as the molecule weight decreases, the activity of chitosan for scavenging of hydroxyl radicals would increase and especially an increase would be seen in its capacity for binding metal ions $[21,22] . \mathrm{Fe}^{+2}$ binding capacity of chitosan comes from amino groups that include free electron couples. $\mathrm{Fe}^{+2}$ binding capacity in low molecule weight is higher when compared to the state of high molecule weight and in addition amino groups of chitosan may do into reaction with free radicals in order to form macro radicals. In other words, hydroxyl and amino groups, which are included in the polymer chain of chitosan, are the structures which form the basic antioxidant feature of chitosan. Besides, it is stated that chitosan forms a barrier against oxygen penetration during film formation and in that way it can prevent oxidation [16, $21,22]$. In a study conducted on the rats that went through hemodialysis, it was found out that chitosan decreased the serum indoxyl sulphate level in $38.5 \%$ and oxidized albumin level in $15.3 \%$ compared to the control group. It is suggested that those results would promote to reduce the stress on the hemodialysis patients and would increase the total plasma antioxidant capacity at the same time [23].

\subsection{Its Usage in Pharmaceutics}

Chitosan has significant usage area in pharmaceutics. Chitosan is a material, which is frequently used in developing especially nasal, vaginal and ophthalmic transdermal, parenteral, colon specific medicines and the medicines taken from human body. Its level of purity, level of acetylation, viscosity and molecular weight are among the qualities to be considered in choosing the chitosan to be used in medicine industry. For that reason, these qualities of the polymer are stated as the qualities to be considered in choosing the appropriate quality chitosan to be used as specific medicine cover agent. The quality of not being soluble in mineral and organic acids of this bio - degradable polymer increases its usage as medicine cover agent in medicine industry [24]. The quality of high level of compliance of chitosan and its low level of toxicity increased its used as auxiliary agent in pharmaceutics especially in recent years. Usage areas of chitosan in pharmaceutics can be listed as follows:

- Usage as diluents agent in compressed medicine.

- Usage as binding agent for wet granulation,

- In tablets and granules of slow release medicine,

- Usage as medicine carrier in micro particle system,

- In the films that control the medicine release,

- In preparation of hydro gels an agent that increases the viscosity in the solution,

- Usage as auxiliary agent for the active ingredients whose dissolution capacity are low in solution,

- Its usage as blender - dispenser,

- Its usage as bio - viscous polymer,

- Its usage for enabling medicine solubility only in specific organs like stomach or colon,

- Its usage for increasing the absorption of the medicine [25].

\subsection{Its Anti - Coagulant Effect}

Heparinization modification is a frequently used method to develop the haemo - compatibility of bio - materials. Usage of chitosan, together with heparinized silk fibroin, has begun to spread recently in order to have the same effect. It was seen that, that composite, which was obtained from chitosan together with heparinized silk fibroin, was seen to produce antimicrobial quality because of the antimicrobial activity of chitosan as well [26]. Especially the chitosan sulfates with low molecule weight show a similar effect with heparin during coagulation. Sulfated chitosan derivatives and 
non - fractional heparin anti - thrombin III form a complex together and accelerate the inactivation of thrombin. Anti coagulant effect of chitosan arises from especially its inhibition of IIa and Xa factors. It is an important component of prothrombinase complex [25, 27].

That coagulant effect of chitosan means that it may coagulate the metals, which show similar effect with chitosan, in drinking water, so that it may be used for drinking water purification. However, it is doubted that chitosan may release some organic substances in its structure during coagulation reactions and for that reason it may increase the disinfection concentration degree, and consequently the toxicity of the water [25].

\subsection{Its Activity as Homoeostatic Agent}

The fact that the chitosan sponge substance, which is derived from poultry larva, has shown better homoeostatic effect compared to gelatin sponge or oxidized cellulose has been proved on rats. Chitosan has that effect by increasing local akaryocyte aggregation, morphologic changes, thrombocyte activation and thrombocyte production. It is stated that chitosan has shown a perfect compatibility with blood and cells, when the bio - compatibility of chitosan is compared with gelatin sponge or oxidized cellulose [28]. With a study, in which the use of chitosan as a homoeostatic agent in the body during the pre - operative period was examined, it was reported that chitosan sponges had a good compatibility with the body like the materials that had been used for that purpose, did not cause any inflammation and also supported neoplasm and vascularity [28].

\subsection{Its Anti- Inflammatory Effect}

With the in vivo and in vitro studies conducted, the anti inflammatory effect of chitosan oligosaccharides with low molecule weight against allergic reactions and allergic asthma was examined and it was found out that chitosan oligosaccharides, which include glucosamine, suppressed the antigen stimulation and cytokine production. It was observed that orally taken chitosan oligosaccharide $(16 \mathrm{mg} / \mathrm{kg} /$ day $)$ reduced IL-4, IL-5, IL-13 and TNF- $\alpha$ mRNA and protein levels. According to those results, it is stated that chitosan may be used as an agent against mast cell mediated allergic inflammatory response and other allergic inflammatory diseases [29]. In a study conducted on rats, it was found out that epigallocatechin-3-gallate (EGCG) triggered the apoptosis of melanoma cells and suppressed the growth of those cells, and when the EGCG given to the rats were covered with a nanoparticle including chitosan, those effects became stronger [30].

\subsection{Its Fat Blocking Quality}

Chitosan does not either digested like dietary fiber and has a lot of positive effects on gastrointestinal system. Chitosan decreases the absorption of bile acids or the cholesterol and thus decreases the blood cholesterol level. In a study conducted on human, it was found out that chitosan, which was consumed 3 - 6 grams for 2 weeks reduced the blood cholesterol level in $6 \%$ and increased the HDL in $10 \%$. It was reported that, chitosan would reduce the absorption of fat consumed with diet in the animals fed with high fat diet, when it was consumed with Vitamin C. However, it was stated that chitosan reduced the absorption of minerals and vitamins soluble in fat together with the absorption of the fat, especially when it was consumed in high amounts. It is considered that the positive load that chitosan molecules have bound the negative loaded molecules like fat and in that way blocks the fat absorption in intestinal and would inhibit the atherosclerotic plaque [25]. In another study conducted on rats, it was found out that chitosan chloride, which was given to rats for 12 weeks and whose de- acetylation level was 0.41 , reduced the tri - glyceride level in $17 \%$ and free fatty acid level in 44\% [31].

\subsection{Its Effect on Regulating Kidney Functions}

In a study conducted, it was found out that total cholesterol of the individuals, who were given $4 \mathrm{~g}$ chitosan per day for 12 weeks and received dialysis treatment dropped $43 \%$ and their kidney function recovered. In another study, it was found out that there was an increase in the blood urea nitrogen and creatine level of the rats after consumption of paraquat, which stimulates the nephro - toxicity; however, among the rats, which were given chitosan oligosaccharide before paraquat, there was no increase in the levels of those parameters. In addition, it was reported that cell deaths occurred in the renal tubules of the rats that were given paraquat, while such case was not observed among the rats, which were given chitosan oligosaccharide before paraquat [32].

\subsection{Its Usage in Weight Control}

In addition to those effects of chitosan in health, it can be used as diet supplement. It is suggested that, this effect of chitosan arises from its fat blocking quality and it binds the negative loaded lipids and reduces their absorption in gastrointestinal system. However, this effect of chitosan has only been observed in the studies conducted on animals and it was reported that such effect had not been observed at the studies conducted on humans [33, 34].

\section{Chitosan Derivatives}

Chitosan is a compound, which can be subject to typical amine reactions. $\mathrm{N}$ - acethylation and Schiff reactions are the two most important amine reactions of chitosan goes through. Chitosan derivatives can easily be produced as the result of those reactions under optimal conditions and chitosan derivatives can be observed as glucan provider compounds [6].

Chitosan is in aldemine form, which is an aldehyde and ketimines form, which is a ketone in room temperature [6]. Chitosan and basic aldehydes form $\mathrm{N}$ - alkyl chitosan through hydrogenation. Existence of component substances with higher or lower molecule weight weakens the hydrogen 
bonds of chitosan. For that reason, $\mathrm{N}$ - Alkyl chitosan remains in water without mixing because of hydrophobic feature of chitosan alkyl bonds. In that way, film forming quality of chitosan can be preserved. Usage areas of other compounds, which are derived from chitosan, are given in Table 2 [6]:

Table 2. Chitosan Derivatives and Their Areas of Usage.

\begin{tabular}{|c|c|}
\hline Chitosan Derivative & Areas of Usage \\
\hline $\begin{array}{l}\text { N - Acetyl Chitosan } \\
\text { (Formyl, acetyl, propionyl, butiryl, hexanoyl, octanoyl, decanoyl, dodecanoyl, tetradecanoyl, lauroyl, } \\
\text { mristoyl, palmitoyl, stearoyl, benzoyl, monochloroacetyl, dichloroacetyl, trifluoroacetyl, carbamoyl, } \\
\text { succinyl, acetoxibenzoyl) }\end{array}$ & $\begin{array}{l}\text { It is used for textile, membrane and medical } \\
\text { purposes. }\end{array}$ \\
\hline $\begin{array}{l}\mathrm{N} \text { - Carboxyalkyl (aryl) Kitosan } \\
\text { (N - Carboxybenzyl, glicin-glucan (N- carboxy-metyl chitosan), alanine glucan, phenylalanine } \\
\text { glucan, tyrosine glucan, serine glucan, glutamic acid glucan, methionine glucan, leucine glucan) }\end{array}$ & $\begin{array}{l}\text { It is used for chromatographic screening and metal } \\
\text { ion gathering. }\end{array}$ \\
\hline $\begin{array}{l}\text { O - Carboxyalkyl Chitosan } \\
\text { (o-Carboxymethyl, cross linked o- Carboxymethyl) }\end{array}$ & $\begin{array}{l}\text { It is used as molecular sieve, viscosity strengthener } \\
\text { and for metal ion gathering. }\end{array}$ \\
\hline $\begin{array}{l}\text { Sugar Derivatives } \\
\text { (1-deoxy-galactic-1-yl-, 1-deoxy-glucide-1-yl-, 1-deoksimelibiit-1-yl-, 1-deoxy-lactide-1-yl-, } 1 \text { - } \\
\text { deoxy-lactide-1-yl-4(2,2,6,6-tetramethylpiperidyne-1-oxyl)-, 1-deoxy-6'-aldehyde- o - lactide -1-yl-, } \\
\text { 1-deoxy-6'-aldehidomelibiit-1-yl-, cellobiit-1-yl - chitosan, products derived from ascorbic acid) }\end{array}$ & It is not used in any specific area yet. \\
\hline $\begin{array}{l}\text { Metal Ion Chelates } \\
\text { (Palladium, copper, silver, iodine) }\end{array}$ & $\begin{array}{l}\text { Catalyst, photography, can be found in the content } \\
\text { of various health products and insecticides. }\end{array}$ \\
\hline $\begin{array}{l}\text { Semi Synthetic Chitosan Resins } \\
\text { (Methyl methacrylate chitosan copolymers, polyurea - urethane, poly (amid ester), acryl amid - } \\
\text { maleic anhydride ) }\end{array}$ & They are the compounds which are used in textile. \\
\hline Natural Polysaccharide Complexes and Types & \\
\hline Chitosan glucans produced in various organisms & $\begin{array}{l}\text { They are involved in flocculation and metal ion } \\
\text { chelation. }\end{array}$ \\
\hline
\end{tabular}

Table 2. (Continued). Chitosan Derivatives and Their Areas of Usage.

\begin{tabular}{|c|c|}
\hline Alkyl chitin and benzyl chitin & Involved in byproduct, serine protease purification. \\
\hline Hydroxy butyl chitin, cyano ethyl chitosan & It is used in desalinization filtration, dialysis and producing isolation papers. \\
\hline Hydroxy ethyl glycol chitosan & Involved in enzyme science, dialysis, production of special papers. \\
\hline Glutaraldehyde chitosan & It has enzyme blocking capacity. \\
\hline
\end{tabular}

\section{Obtaining Chitosan and Its Storage Conditions}

Chitosan, which is obtained from shellfish especially as a food waste, is a product that can be economically obtained. It is important especially for recycling of carotenoids, which are included in the hard shell parts of shellfish in high amounts. Those carotenoids obtained from shells can be used as a preservative for the storage period or protecting gel consistency of fish, especially for salmon [25].

$6.3 \mathrm{~kg}$ of hydrogen chloride, $1.8 \mathrm{~kg}$ of sodium hydroxide together with nitrogen, 0.5 liter service water and 0.9 liter cooling water are required in order to obtain $1 \mathrm{~kg}$ of $70 \%$ de - acetylated chitosan from shellfish. The factor that especially increases the costs of chitosan in a high level is the use of work power and it changes according to the country. Chitin and chitosan are produced in high amounts in the countries where shellfish are consumed in high amounts like India, Japan, Poland, Norway and Australia [35].

\section{Chitosan from the View of Health Authorities}

Chitosan is a compound, which has been used as an food additive since 1983, in Japan and it is accepted as a molecule with low toxicity in India, Japan, Poland, Norway and Australia. Chitosan has been used as a condenser substance in meat products since 2007. It was examined by Codex Alimentarius Commission in 2003. However, it is not included in General Standards in Food Additives list yet. The European Food Safety Authority (EFSA) stated that chitosan had an active character in enabling normal LDL levels in blood. In addition, the use of chitosan, which is produced from prawn, in food and drink applications was approved in GRAS status in 2001; however, it was withdrawn later. However, it is possible that the chitosan produced from prawn would be taken into GRAS status again. The use of chitosan, which is produced from $A$. niger, in the production of alcoholic drinks as secondary direct additive in an amount of 10-500 g/100 L is accepted in GRAS status [9, 22, 34].

Side Effects: Although there is no long term study on 
humans regarding chitosan, it has been reported that chitosan may suppress the absorption of vitamins and minerals that are dissolved in fat. For that reason, the use of chitosan by people with intestinal malabsorption is not recommended. It is believed that the changes triggered by chitosan in intestinal flora are generally useful. However, how those effects would change in long term is not known. Depending on the limited number of studies on that subject, it si recommended that the patients are given $2.4 \mathrm{~g}$ chitosan per day in order to increase the anticoagulant effect of warfarin [25].

Therapeutic Dose: As reported in many human studies, it is stated that it can be consumed in an amount of $3-6$ grams per day with food [25].

Toxicity: Chitosan is a compound, whose toxic quality is extremely low.

\section{Conclusion and Recommendations}

Chitosan, which has especially been used in medicine industry for a long time, is a product recently been used in many other areas. When its use in food industry is considered, being a good antimicrobial agent and enabling the extension of storage period or shelf lives of food are among its qualities that are beginning to be used in recent years. It is no doubt that the reason for the spread of use of chitosan is that it has proven toxic effect on human and it is a compound included in GRAS list in some countries for years. For that reason, it is thought that chitosan can securely be used in health area and food sector because no toxic effect of chitosan has been defined so far. In addition, with the chitosan, which is especially produced from shellfish, it is thought that the common use of chitosan, which causes environmental pollution in large scale and constitute waste load in big amounts, would be useful in many areas. However, it should not be forgotten that there is a lack of long term study conducted on human and we think that there is a need for studies to fill that gap.

\section{References}

[1] Hafdani, F.N. and N. Sadeghinia, A review on application of chitosan as a natural antimicrobial. World Academy of Science, Engineering and Technology, 2011. 74: p. 257-261.

[2] Alishahi, A., Chitosan: A Bioactive Polysaccharide in MarineBased Foods. Biochemistry, Genetics and Molecular Biology » "The Complex World of Polysaccharides", ed. D.N. Karunaratne. 2012: CC BY 3.0 license. 409-428.

[3] de Britto, D., et al., Quaternary salts of chitosan: History, antimicrobial features, and Prospects. International Journal of Carbohydrate Chemistry, 2011. 2011.

[4] Uçan, F. and H.A. Mercimek, Gıda Endüstrisinde Kitosan Filmlerin Önemi. Gida Bilim ve Teknoloji Dergisi, 2013. 1(2): p. 79-85.

[5] İmamoğlu, Ö., Biyokontrolde doğal ürünlerin kullanılması; Kitosan. Türk Hijyen ve Deneysel Biyoloji Dergisi, 2011. 68(4): p. 215-222.
[6] Ravi Kumar, M.N., A review of chitin and chitosan applications. Reactive and functional polymers, 2000. 46(1): p. $1-27$.

[7] de Alvarenga, E.S., Characterization and properties of chitosan. Biotechnology of biopolymers, 2011: p. 91-108.

[8] Liu, N., et al., Effect of MW and concentration of chitosan on antibacterial activity of Escherichia coli. Carbohydrate polymers, 2006. 64(1): p. 60-65.

[9] Klinkesorn, U., The Role of Chitosan in Emulsion Formation and Stabilization. Food Reviews International, 2013. 29(4): p. 371-393.

[10] Sashiwa, H. and S.-i. Aiba, Chemically modified chitin and chitosan as biomaterials. Progress in Polymer Science, 2004. 29(9): p. 887-908.

[11] Qin, C., et al., Water-solubility of chitosan and its antimicrobial activity. Carbohydrate polymers, 2006. 63(3): p. 367-374.

[12] Alishahi, A. and M. Aïder, Applications of chitosan in the seafood industry and aquaculture: a review. Food and Bioprocess Technology, 2012. 5(3): p. 817-830.

[13] Kong, M., et al., Antimicrobial properties of chitosan and mode of action: A state of the art review. International Journal of Food Microbiology, 2010. 144(1): p. 51-63.

[14] Fernandez-Saiz, P., et al., Effects of chitosan films on the growth of Listeria monocytogenes, Staphylococcus aureus and Salmonella spp. in laboratory media and in fish soup. International journal of food microbiology, 2010. 137(2): p. 287-294.

[15] Benhabiles, M., et al., Antibacterial activity of chitin, chitosan and its oligomers prepared from shrimp shell waste. Food Hydrocolloids, 2012. 29(1): p. 48-56.

[16] Aider, M., Chitosan application for active bio-based films production and potential in the food industry: Review. LWTFood Science and Technology, 2010. 43(6): p. 837-842.

[17] Ojagh, S.M., et al., Effect of chitosan coatings enriched with cinnamon oil on the quality of refrigerated rainbow trout. Food Chemistry, 2010. 120(1): p. 193-198.

[18] Ojagh, S.M., M. Rezaei, and S.H. Razavi, Improvement of the Storage Quality of Frozen Rainbow Trout by Chitosan Coating Incorporated with Cinnamon Oil. Journal of Aquatic Food Product Technology, 2014. 23(2): p. 146-154.

[19] Poverenov, E., et al., Effects of a composite chitosan-gelatin edible coating on postharvest quality and storability of red bell peppers. Postharvest Biology and Technology, 2014. 96: p. 106-109.

[20] Kamil, J.Y., Y.-J. Jeon, and F. Shahidi, Antioxidative activity of chitosans of different viscosity in cooked comminuted flesh of herring (Clupea harengus). Food Chemistry, 2002. 79(1): p. 69-77.

[21] Feng, T., et al., Enhancement of antioxidant activity of chitosan by irradiation. Carbohydrate Polymers, 2008. 73(1): p. 126-132.

[22] No, H., et al., Applications of chitosan for improvement of quality and shelf life of foods: a review. Journal of food science, 2007. 72(5): p. R87-R100. 
[23] Anraku, M., et al., Effects of chitosan on oxidative stress and related factors in hemodialysis patients. Carbohydrate Polymers, 2014.

[24] Balamurugan, M., Chitosan: A perfect polymer used in fabricating gene delivery and novel drug delivery systems. International Journal of Pharmacy \& Pharmaceutical Sciences, 2012. 4(3).

[25] Gavhane, Y., A. Gurav, and A. Yadav, Chitosan and Its Applications: A Review of Literature. Int. J. Res. Pharma. Biomedical Sci, 2013. 4: p. 311-331.

[26] Wang, J., et al., Dual-functional composite with anticoagulant and antibacterial properties based on heparinized silk fibroin and chitosan. Colloids and Surfaces B: Biointerfaces, 2011. 85(2): p. 241-247.

[27] Vikhoreva, G., et al., Preparation and anticoagulant activity of a low-molecular-weight sulfated chitosan. Carbohydrate Polymers, 2005. 62(4): p. 327-332.

[28] Gu, R., et al., The performance of a fly-larva shell-derived chitosan sponge as an absorbable surgical hemostatic agent. Biomaterials, 2010. 31(6): p. 1270-1277.

[29] Chung, M.J., J.K. Park, and Y.I. Park, Anti-inflammatory effects of low-molecular weight chitosan oligosaccharides in IgE-antigen complex-stimulated RBL-2H3 cells and asthma model mice. International immunopharmacology, 2012. 12(2): p. 453-459.
[30] Siddiqui, I.A., et al., Excellent anti-proliferative and proapoptotic effects of (-)-epigallocatechin-3-gallate encapsulated in chitosan nanoparticles on human melanoma cell growth both in vitro and in vivo. Nanomedicine: Nanotechnology, Biology and Medicine, 2014.

[31] Liu, X., et al., Effect of chitosan, O-carboxymethyl chitosan, and $\mathrm{N}-[(2$-hydroxy-3-N, N-dimethylhexadecyl ammonium) propyl] chitosan chloride on overweight and insulin resistance in a murine diet-induced obesity. Journal of agricultural and food chemistry, 2012. 60(13): p. 3471-3476.

[32] Yoon, S.P., et al., Protective effects of chitosan oligosaccharide on paraquat-induced nephrotoxicity in rats. Food and Chemical Toxicology, 2011. 49(8): p. 1828-1833.

[33] Jull, A.B., et al., Chitosan for overweight or obesity. Cochrane Database Syst Rev, 2008. 3.

[34] EFSA, E.F.S.A., Chitosan related health claims. EFSA Journal, 2011. 9: p. 2214-2234.

[35] Bostan, K., T. Aldemir, and A. Aydın, Kitosan ve antimikrobiyal aktivitesi. Türk Mikrobiyoloji Cemiyeti Dergisi, 2007. 37(2): p. 118-127. 